\title{
Immersive Open Surgery Simulation
}

\author{
Ali Al-khalifah, Rachel McCrindle, and Vassil Alexandrov \\ School of Systems Engineering, The University of Reading, Whiteknights PO Box 225, \\ Reading RG6 6AY, UK \\ \{a.h.al-khalifah, r.j.mccrindle, v.n.alexandrov\}@rdg.ac.uk \\ http://www.sse.rdg.ac.uk
}

\begin{abstract}
Realistic medical simulation has great potential for augmenting or complimenting traditional medical training or surgery planning, and Virtual Reality (VR) is a key enabling technology for delivering this goal. Although, medical simulators are now widely used in medical institutions, the majority of them are still reliant on desktop monitor displays, and many are restricted in their modelling capability to minimally invasive or endoscopic surgery scenarios. Whilst useful, such models lack the realism and interaction of the operating theatre. In this paper, we describe how we are advancing the technology by simulating open surgery procedures in an Immersive Projection Display CAVE environment thereby enabling medical practitioners to interact with their virtual patients in a more realistic manner.
\end{abstract}

\section{Introduction}

Virtual reality (VR) is a key technology for enabling medical practitioners to visualize and interact with their models/datasets. Indeed, the potential for VR as a modelling and simulation tool for medical training or surgery planning has already been demonstrated through a number of research-based and commercial simulation systems $[1,2,3]$. However, the majority of these systems are desktop based applications and hence lack the presence, immersion, interactive and collaborative elements possible from developing VR applications within a CAVE environment. Additionally, with the exception of the US Department of Defence who are developing an immersive trauma related military medical training system [4], the majority of these applications have been restricted to modelling specific endoscopic surgical procedures.

Simulation of open surgical procedures presents a new set of challenges for researchers and developers. These challenges exist due to the complex procedures that must be modelled such as tissue deformation, suturing, incisions and bleeding of the patient, as well as the delicate movements and responses of the surgeon's hand. The nature of these procedures mean that open surgery simulations cannot be as realistically modelled on a desktop display as can an endoscopic surgical procedure.

In this paper we describe our initial work in simulating an open surgical procedure within a CAVE based environment. 


\section{The Application}

The aim of our virtual immersive application is to simulate the real-life scenario of an operating theatre with a patient lying on the operating table surrounded by surgeons and other medical staff. Through our modelling of this scenario, users of the system can surround the virtual patient and interact with it in the same manner as that of a real surgical team interacting with a real patient.

To achieve this we have initially focussed on two types of modelling procedures surface deformations and model/object manipulations. Using a 3-D wand, the application enables the user to arbitrarily deform a pre-defined surface, the abdomen in this case, such that the virtual body can be cut open and the internal organ structures - stomach, liver, kidneys, small intestine, and the heart - exposed ready for medical intervention. Through this approach realism is added to the model as the elastic tissue of the human body can be stretched or squeezed when it come into contact with other objects or structures. This is an important characteristic for an open surgery medical simulation application.

\section{Environment and Implementation}

The CAVE used for the simulation is a large box shaped display device composed of 4 flat screens (three walls and a floor) approximately 10x10x10 foot in dimensions. Five to six users may step inside the CAVE space at any one time and further collaboration may also occur with other CAVE based users across a network. Stereoscopic images are back projected onto the screen by special projectors. In order for users to see the images inside the CAVE as 3D objects special liquid crystal shutter glasses are worn. Interaction with the displayed model is via a head tracking device and a 3D 6 degree of freedom mouse known as a 'wand'. In this application the wand simulates scalpel interaction.

The application is coded in the $\mathrm{C}$ language using the popular graphics interface OpenGL and CAVElib. OpenGL is used to model all surface and deformable objects as well as other scene aspects such as light and material properties. CAVElib is used to implement the environment interaction and for routines such as tracking, navigation, and dynamic object manipulation. The software tools were selected on the basis of their availability, ease of use and compatibility with most operating platforms. Organ models are imported into the model as individual data files, converted from various formats such as 3D-Studio (.3ds), VRML (.wrl) and SGI-Inventor (.iv) using Deep Exploration [5].

\section{Results}

As can be seen from Figure 1 the developed application allows us to simulate a collaborative operating environment as well as simple tissue deformation and manipulation operations and exposure of the organs pertinent to the surgical procedure being conducted (Fig 2a and 2b). Object and tool manipulations are also implemented that enable the user to interact with static non-deformable models such as surgical tools. 


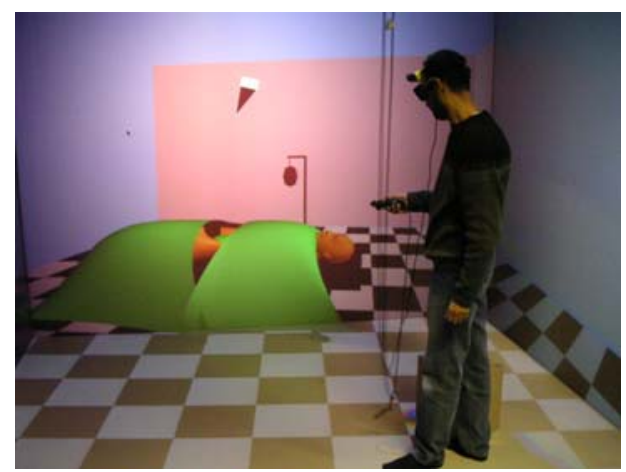

Fig. 1. Surgeon Interacting with the Virtual Patient
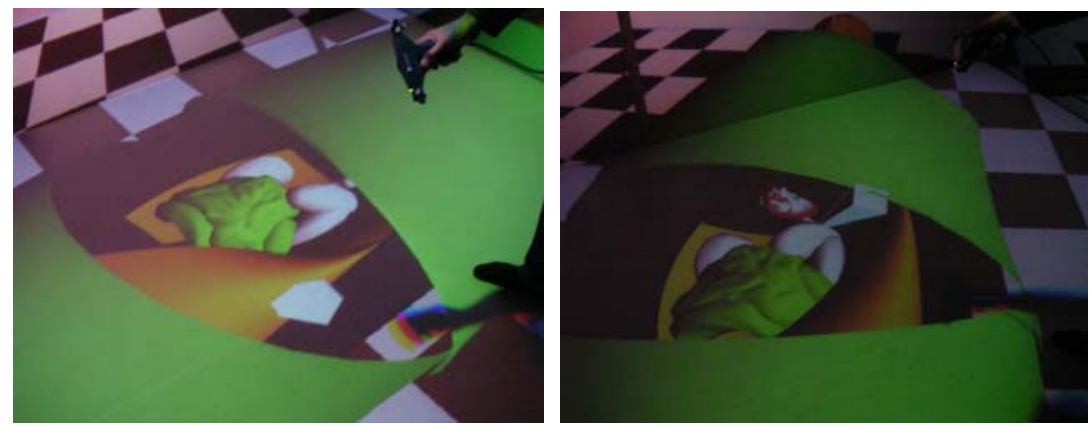

Fig. 2a and $\mathbf{2 b}$. The abdominal is opened by deforming its surface and internal organs displayed ready for interventions

\section{Evaluation}

In order to gain feedback on our simulations and to ascertain views of practitioners on the applicability of virtual reality for medical training and surgery planning, a study, conducted via questionnaires and CAVE-based demonstrations, is underway. Initial results are encouraging as part-shown in Tables 1 and 2; based on feedback from 30 participants including medical consultants, general practitioners, researchers, surgeons, and medical educators.

Table 1. Initial feedback results in Percentages (Part 1)

\begin{tabular}{|c|c|c|c|}
\hline Survey Question & Agree & Not Sure & Disagree \\
\hline $\begin{array}{l}\text { Medical practitioners will eventually benefit from the immersive } \\
\text { simulation technology }\end{array}$ & 41 & 28 & 31 \\
\hline $\begin{array}{l}\text { Immersive displays such as the CAVE provide more powerful tools and } \\
\text { features to the medical profession than a desktop display }\end{array}$ & 47 & 24 & 29 \\
\hline $\begin{array}{l}\text { The CAVE can be a potential medium for future medical open surgery } \\
\text { simulations }\end{array}$ & 26 & 52 & 22 \\
\hline I would use this technology to simulate open interventions & 23 & 57 & 20 \\
\hline
\end{tabular}


However, whilst the results show a general appreciation and acceptance of VR simulations, we have found that visual simulation alone may not be adequate to convince the medical community of the applicability of VR technology in meeting their full expectations. For example, a haptic interface will be a major enhancement to open surgery simulation adding further realism and enhancing interactions with the environment and patient.

Table 2. Initial feedback results in Percentages (Part 2)

\begin{tabular}{|l|c|c|c|}
\hline \multicolumn{1}{|c|}{ Survey Question } & High & Neutral & Low \\
\hline Rate the usefulness of the CAVE in particular for medical simulations & 41 & 12 & 47 \\
\hline Rate the usefulness of VR in general for medical simulations & 53 & 22 & 25 \\
\hline How far would you like to see immersive CAVE technology & 43 & 34 & 23 \\
\hline
\end{tabular}

\section{Conclusions and Further Work}

We have successfully implemented a simple immersive application that simulates an open surgery procedure, allowing the user to open the abdominal cavity, observe deformation of elastic tissue, and to interact with internal organs. This type of surgery is hugely demanding in terms of computer power and complexity of operations involved and hence this application barely scratches the surface of such complex applications. However, it gives us a strong basis on which to develop further complex procedures and operations. The major advantage of this application is that through exploiting the CAVE technology, it supports the crucial elements associated with open surgery operations such as, immersion, interactivity, presence, collaboration and multi-user interactions. Initial feedback from practitioners involved in medical education and surgery planning has been encouraging and generally positive.

We will continue to develop the complexity of our open surgery models and simulations. Collaboration between users located at geographically different locations is being further developed. Haptic rendering inside the CAVE is another area of interest that when implemented will greatly improve the realism of open surgery simulations.

\section{References}

1. Liu A., Tendick F., Cleary K., Kaufmann C.: Survey of Surgical Simulation: Applications, Technology, \& Education. Presence: Teleoperators \& Virtual Env. (2003), 599-614

2. Hollands R. J., Trowbridge E. A. A virtual reality training tool for the arthroscopic treatment of knee disabilities. University of Sheffield, UK (2005)

3. Vidal F. P., Bello F., Brodlie K., John N. W., Gould D., Phillips R., Avis N. J. Principles and Applications of Medical Virtual Environments. Eurographics 2004 State of the Art reports (2004)

4. Immersion Corporation. http://immr.client.shareholder.com/ReleaseDetail.cfm?ReleaseID= 111797 (2005)

5. Right Hemisphere. http://www.righthemisphere.com/ (2005) 